\title{
THE FREE LIE RING AND LIE REPRESENTATIONS OF THE FULL LINEAR GROUP
}

BY

\author{
ANGELINE J. BRANDT
}

Introduction. The present paper is a continuation and amplification of R. M. Thrall's paper On symmetrized Kronecker powers and the structure of the free Lie ring [7](1), which we shall denote as FR. The notation used in FR shall be adopted in this paper. The author wishes to express her appreciation to Professor R. M. Thrall of the University of Michigan, who suggested the problem of the present paper and gave valuable guidance during the preparation of it.

We propose to study the structure of the free Lie ring, its characteristic ideals, and certain related right ideals in the group ring of the symmetric group. We operate over a field $K$ of characteristic zero and thus insure that the named group ring be semi-simple (which is not the case for fields of characteristic $p$ ). In FR a recursion formula $[7$, p. 386] was developed from which the irreducible constituents of the $m$ th Lie representation for $m \leqq 10$ were obtained. The main result of the present investigation is Theorem III ( $\$ 2)$ which gives a direct formula for the character of the $m$ th Lie representation.

1. The free Lie ring and Lie representations of the full linear group. The free non-associative $K$ ring $Z_{n}$, the free Lie ring $L=L_{n}$, and the $m$ th Lie representation are defined in FR [7, pp. 372-373]. It is convenient to make the definition of ideal more explicit than in FR. We begin with the concept of a homomorphism of a ring $Z_{n}$ onto a ring $Z_{n}{ }^{*}$, and then call a subset $J$ of $Z_{n}$ an ideal if it is the kernel of some homomorphism. A homogeneous ideal is defined in FR $[7$, p. 372]. The following result was stated without proof in FR [7, p. 372].

THEOREM I. If $\sigma$ is a homomorphic mapping of $Z_{n}$ onto $Z_{n}^{*}$ such that $\sigma\left(Z^{m}\right)=Z^{*^{m}}$ and with a homogeneous ideal $J$ as kernel, then $Z_{n}^{*}=\sum Z^{*^{m}}$ (direct sum).

Proof. It is obvious that $Z_{n}^{*}=\sum Z^{*^{m}}$ but we need to show that it is a direct sum. Suppose $Z_{n}{ }^{*}$ is not the direct sum of $Z^{* m}$ s. Then for some $m$ there is an element $z^{m}$ of the module $Z^{m}$ for which $\sigma\left(z^{m}\right)=\sigma\left(z^{m-1}+z^{m-2}+\cdots\right)$ $\neq 0$ where $z^{i}$ denotes some element of $Z^{i}$. By homomorphism properties $\sigma\left[z^{m}-\left(z^{m-1}+z^{m-2}+\cdots\right)\right]=0$, that is, $z^{m}-\left(z^{m-1}+z^{m-2}+\cdots\right) \in J$ and hence $z^{m} \in J$ and so $\sigma\left(z^{m}\right)=0$, contrary to our assumption. Thus a contradic-

Presented to the Society, November 27, 1943 ; received by the editors April 8, 1944.

(1) Numbers in brackets refer to the Bibliography at the end of the paper. 
tion has been reached by assuming that $Z_{n}^{*}$ is not the direct sum of $Z^{* m}$ 's and hence $Z_{n}^{*}=\sum Z^{*^{m}}$ (direct sum).

Denote the $n$ generators of the free Lie ring by $r_{1}, \cdots, r_{n}$. When dealing with non-associative multiplication some means of inserting parentheses must be indicated. In particular, denote the product $\left(\cdots\left(\left(r_{i_{1}} \circ r_{i_{2}}\right) \cdots r_{i_{n-1}}\right) r_{i_{n}}\right)$ by $r_{i_{1}} \cdots_{i_{n-1} i_{n}}$ and call this particular way of multiplying the right normed product. For example, $r_{12345}=\left(\left(\left(r_{1} \circ r_{2}\right) r_{3}\right) r_{4}\right) r_{5}$. Magnus $[6$, p. 106] shows that right normed products span the whole ring. However, these right normed products are not linearly independent and one of our purposes is to obtain relations between these right normed products. A monomial in the $r$ 's we denote by $f_{i}^{\prime}(g)=f_{i}^{r}\left(r_{g_{1}}, \cdots, r_{g_{r}}\right)$, where $g=\left(g_{1}, g_{2}, \cdots, g_{r}\right)$ denotes one of the $n^{r}$ ordered sets of $r$ indices chosen from $1, \cdots, n$, repetitions permitted. Any linear combination of these monomials is an element of the free Lie ring. For a certain $r, f_{i}^{T}(g)$ form basis elements of a $K$ vector space or module $L^{r}$. By Theorem I, $L=L^{1}+L^{2}+\cdots$. The module $L^{m}$ has dimension $\left({ }^{2}\right) \psi_{m}(n)$ $=(1 / m) \sum \mu(m / d) n^{d}$, where $d$ runs over all divisors of $m$.

The mapping $z_{h} \rightarrow z_{h}^{\prime}=\sum a_{h k} z_{k}$ where $A=\left\|a_{h k}\right\|$ is any nonsingular $n$-rowed matrix with elements in the field $K$ is called a linear automorphism of the ring $Z_{n}$. A representation of power type of $A$ is defined in FR [7, p. 373]. One such representation is the particular representation afforded by the transformation in the module $L^{m}$ induced by $A$. This is called the mth Lie representation.

Ideals which are invariant under all automorphisms are called characteristic ideals.

THEOREM II. Every characteristic ideal I of the free Lie ring can be expressed as the direct sum $I \cap L^{1}+I \cap L^{2}+\ldots$ ( $\cap$ denotes common part), that is, every characteristic ideal of $L$ is a homogeneous ideal [7, p. 371].

Proof. $L=L^{1}+L^{2}+\cdots$. Let $a$ be an element of $I$ composed of compo. nents $a_{i}$ from each $L^{i}$, that is, $a=a_{1}+a_{2}+\cdots+a_{r}$. Apply the linear automorphisms $A_{1}, A_{2}, \cdots, A_{\text {s }}$ to $a$.

$$
\begin{aligned}
& A_{1}(a)=L_{1}\left(A_{1}\right) a_{1}+L_{2}\left(A_{1}\right) a_{2}+\cdots+L_{r}\left(A_{1}\right) a_{r}, \\
& A_{2}(a)=L_{1}\left(A_{2}\right) a_{1}+L_{2}\left(A_{2}\right) a_{2}+\cdots+L_{r}\left(A_{2}\right) a_{r} \text {, } \\
& A_{s}(\dot{a})=L_{1}\left(\dot{A}_{s}\right) a_{1}+L_{2}\left(\dot{A}_{s}\right) a_{2}+\cdots+L_{r}\left(\dot{A}_{8}\right) a_{r} .
\end{aligned}
$$

Taking a linear $k$ combination, $b=\sum_{i=1}^{s} \alpha_{l} A_{i}(a)=\sum_{j=1}^{r} \sum_{i=1}^{s} \alpha_{l} L_{j}\left(A_{i}\right) a_{j}$ for any element $\alpha_{i}$ in $k$. Since $I$ is a characteristic ideal, each $A_{i}(a)$ and hence also the linear combination $b$ lies in $I$. Clearly, $I \supseteq I \cap L^{1}+I \cap L^{2}+\cdots$. To show that $I \cap L^{1}+I \cap L^{2}+\cdots \supseteq I$, choose $A_{1} \cdots A_{q}$ and $\alpha_{1} \cdots \alpha_{q}$

(2) We give a calculation for this dimension later in the paper. 
so that $\sum_{i=1}^{s} \alpha_{i} L_{j}\left(A_{i}\right)=0$ if $j \neq c$ and equals 1 if $j=c\left(^{3}\right)$. Thus $\sum_{i=1}^{s} \alpha_{i} A_{i}(a)$ $=\sum_{i=1}^{s} \alpha_{t} L_{j}\left(A_{t}\right) a_{c}=a_{c}$ if $j=c$ and equals 0 if $j \neq c$. Hence each $a_{c}$ is in $I$ and thus in $I \cap L_{c}$. The $c$ may take on the values which appear in the components of $a$. Therefore $I \cap L^{1}+I \cap L^{2}+\cdots \supseteq I$ and since $I \supseteq I \cap L^{1}+I \cap L^{2}$ $+\cdots$, it follows that $I \cap L^{1}+I \cap L^{2}+\cdots=I$. Since $L$ is a direct sum of the $L^{i}$ 's the sum on the left will be a direct sum.

2. The character of the $m$ th Lie representation. Let $L_{m}(A)$ denote the matrix describing the transformation in the module $L^{m}$ and let $[m]$ denote the character (that is, trace) of $L_{m}(A)$. Let $(\alpha)=\left(\alpha_{1}, \cdots, \alpha_{m}\right)$ denote the partition $\alpha_{1}+2 \alpha_{2}+\cdots+m \alpha_{m}=m$ of $m$ and let $\chi_{(\alpha)}^{(\lambda)}$ denote the value for the class $(\alpha)$ of the character of the symmetric group defined by $(\lambda)$. Then let $\{\lambda\}$ be the corresponding irreducible character of the full linear group. The following main theorem gives a direct formula for the character of the $m$ th Lie representation.

THEOREM III. $[m]=1 / m \sum \mu(d) s_{d}^{m / d}$, where $d$ ranges over all divisors of $m$. $\mu(d)$ is the Möbius function( $\left.{ }^{4}\right)$ and $s_{d}$ is the trace of $A^{d}, A$ being an arbitrary element of the full linear group.

Let $t_{1}\left(g ; s_{1} \cdots s_{g}\right)=t_{1}(g ; s)=1 / g \sum_{\left.d\right|_{g} \mu}(d) s_{d}^{g / d}$ and let $t_{h}\left(g ; s_{h} \cdots s_{g h}\right)$ $=t_{h}(g ; s)=1 / g \sum_{\left.d\right|_{g} \mu}(d) s_{h d}^{g / d}$ ( $g$ is any integer) since if $t_{1}(g ; s)$ is the trace of the matrix $B$ in some representation $A \rightarrow B(A)$, then $t_{h}(g ; s)=\operatorname{tr}\left(B^{h}\right)$ in that representation. For since $\operatorname{tr} B(A)=t_{1}\left(g ; s_{1} \cdots s_{0}\right), \operatorname{tr}[B(A)]^{h}=\operatorname{tr} B\left(A^{h}\right)$ $=t_{h}\left(g ; s_{h}, s_{2 h}, \cdots, s_{g h}\right)=t_{h}(g ; s)$. We wish to show that $t_{1}(g ; s)=\operatorname{tr} L_{g}(A)$. It will then follow that $t_{h}(g ; s)=\operatorname{tr} L_{g}\left(A^{h}\right)=\operatorname{tr}\left(L_{g}(A)\right)^{h}$. If $(\alpha)$ is a partition of $m$, we set $\phi(\alpha)=q_{\alpha_{1}}\left(t(1 ; s) q_{\alpha_{2}}(t(2 ; s)) \cdots q_{\alpha_{m}}(t(m ; s))\right.$, where [2, p. 106] $\left.q_{r}(t(g ; s))=q_{r}\left(t_{1}(g ; s)\right), t_{2}(g ; s), \cdots, t_{r}(g ; s)\right)=\sum^{2}\left(1 / \nu_{1} ! \cdots \nu_{r} !\right)\left(t_{1}(g ; s) / 1\right)^{\nu_{1}}$ $\cdots\left(t_{r}(g ; s) / r\right)^{\nu_{r}}$ and $\nu_{1}+2 \nu_{2}+\cdots+r \nu_{r}=r$.

\section{LEMMA I. $\sum_{\alpha} \phi(\alpha)=s_{1}^{m}$.}

Suppose $\sum \phi(\beta)=s_{1}^{\mu}$ for all $\mu<m$, where the summation is over all partitions $(\beta)$ of $\mu$. It is sufficient to establish the lemma if we show that we obtain an equality by differentiating each side of $\sum_{(\beta) \text { of } \mu} \phi(\beta)=s_{1}^{\mu}$ with respect to each $s_{k}$ when $\mu=m$, since the polynomials involved are isobaric and clearly identical if all of their partial derivatives are equal.

From the manner in which the $t_{h}(g ; s)$ 's were defined, it is clear that $s_{k}$ appears in $t_{h}(g ; s)$ only if $h \mid k$ and $k / h \mid g$, that is, $k \mid g h$.

Differentiating a particular $t_{h}(g ; s)$ with respect to $s_{k}$, we obtain

(3) The possibility of such a choice is shown by Weyl [4, pp. 93 and 130] for irreducible constituents of the full linear group arising from the decomposition of tensor space. It is known by $[7$, pp. 384-385] that the irreducible constituents of the Lie representation are equivalent to those that come from the decomposition of tensor space.

(4) This is the familiar Möbius function. Its properties are discussed in [3, pp. 109-112]. 


$$
\left(\partial / \partial s_{k}\right) t_{h}(g ; s)=(1 / d) \mu(d) s_{h d}^{(o / d)-1}=(h / k) \mu(k / h) s_{k}^{o /(k / h)-1}
$$

if $k / h$ and $g h / k$ are integers; otherwise $\left(\partial / \partial s_{k}\right) t_{h}(g ; s)=0$. Since $h$ will be some definite number and $k$ is fixed, $k / h \mid g$ gives just one term involving $s_{k}$.

$$
\frac{\partial}{\partial s_{k}} q_{r}(t(g ; s))=\sum_{h=1}^{r} \frac{\partial q_{r}(t(g ; s))}{\partial t_{h}(g ; s)} \cdot \frac{\partial t_{h}(g ; s)}{\partial s_{k}} .
$$

But [2, p. 107]

$$
\partial q_{r}\left(t(g ; s) / \partial t_{h}(g ; s)=(1 / h) q_{r-h}(t(g ; s)) .\right.
$$

Substituting (1) and (3) in (2), we have

$$
\begin{aligned}
\frac{\partial}{\partial s_{k}} q_{r}(t(g ; s)) & =\sum_{h}(1 / h) q_{r-h}(t(g ; s))(h / k) \mu(k / h) s_{k}^{o /(k / h)-1} \\
& =1 / k \sum_{h} q_{r-h}(t(g ; s)) \mu(k / h) s_{k}^{(g h / k)-1},
\end{aligned}
$$

where we sum over all $h$ for which $k / h$ and $g h / k$ are integers.

For a partition $(\alpha)$ of $m$, differentiating the function $\phi(\alpha)=q_{\alpha_{1}}(t(1 ; s))$ $\cdots q_{\alpha_{m}}(t(m ; s))$ with respect to $s_{k}$, we have

$$
\begin{aligned}
\frac{\partial \phi(\alpha)}{\partial s_{k}}= & \sum_{g=1}^{m} q_{\alpha_{1}}(t(1 ; s)) q_{\alpha_{2}}(t(2 ; s)) \cdots \frac{\partial q_{\alpha_{o}} t(g ; s)}{\partial s_{k}} \cdots q_{\alpha_{m}}(t(m ; s)) \\
= & 1 / k \sum_{o=1}^{m} q_{\alpha_{1}}(t(1 ; s)) q_{\alpha_{2}}(t(2 ; s)) \\
& \cdots \sum_{h} q_{\alpha_{g}-h}(t(g ; s)) \mu(k / h) s_{k}^{(g h / k)-1} \cdots q_{\alpha_{m}}(t(m ; s)),
\end{aligned}
$$

where $k / h$ and $g h / k$ are integers.

Considering the partial differentiation of $\sum \phi(\alpha)$, a partition $(\beta)$ of $m-g h$ is taken. By adding $h$ to $\beta_{g}$ a partition $(\alpha)$ of $m$ is obtained, that is, $\left(\beta_{1}, \cdots, \beta_{g}, \cdots, \beta_{m}\right)=\left(\alpha_{1}, \cdots, \alpha_{g}-h, \cdots, \alpha_{m}\right)$. Therefore as $(\beta)$ runs over all partitions of $m-g h,(\alpha)$ runs over all partitions of $m$ where $\alpha_{g} \geqq h$. If $\alpha_{g}<h, q_{\alpha_{g}-h}(t(g ; s))=0$; if $\alpha_{g}=h, q_{0}(t(g ; s))=1$. Therefore

$$
\frac{\partial}{\partial s_{k}} \sum_{(\alpha)} \phi(\alpha)=1 / k \sum_{o=1}^{m} \sum_{h}\left(\sum \phi(\beta) \mu(k / h) s_{k}^{(o h / k)-1}\right),
$$

where the inner summation is over all partitions $(\beta)$ of $m-g h$. The change in order of summation on the right does not affect the result since the summations are over finite quantities.

By our induction hypothesis $\sum_{(\beta) \text { of } m-g h} \phi(\beta)=s_{1}^{m-g h}$; therefore

$$
\frac{\partial}{\partial s_{k}} \sum_{(\alpha)} \phi(\alpha)=1 / k \sum_{g=1}^{m}\left(\sum_{h} s_{1}^{m-g h} \mu(k / h) s_{k}^{(g h / k)-1}\right),
$$


where $k / h$ and $g h / k$ are integers. Here $k$ is fixed for any particular differentiation and $k \mid g h$. Let $g h / k=p$. Take all $h$ and all $g$ for which $k / h$ and $p$ are integers. Since $h|k, p| g$. For each divisor $h$ of $k$ take $g=p k / h$ and for each value of $h$, a term $s_{1}^{m-k p} s_{\mathbf{k}}^{p-1}$ with coefficient $1 / k \mu(k / h)$ is obtained. Therefore

$$
\frac{\partial}{\partial s_{k}} \sum_{(\alpha)} \phi(\alpha)=1 / k \sum_{p=1}^{[m / k]}\left[\sum \mu(k / h) s_{1}^{m-k p} s_{k}^{p-1}\right]
$$

where for each $p, h$ runs over all divisors of $k$. For $k \neq 1$, the result is zero by the properties of the Möbius function. For $k=1$, we have

$$
\frac{\partial}{\partial s_{k}} \sum_{(\alpha)} \phi(\alpha)=\sum_{p=1}^{m} \mu(1) s_{1}^{m-p} s_{1}^{p-1}=m s_{1}^{m-1},
$$

which completes the proof of the lemma.

Denote by $A[\alpha]$ the matrix $S_{\alpha_{1}}\left(L_{1}(A)\right) \times S_{\alpha_{2}}\left(L_{2}(A)\right) \times \cdots$ and set $[(\alpha)]=\operatorname{tr}[A]=q_{\alpha_{1}}\left(\operatorname{tr} L_{1}(A)\right) q_{\alpha_{2}}\left(\operatorname{tr} L_{2}(A)\right) \cdots$, where $q_{r}\left(\operatorname{tr} L_{i}(A)\right)$ means $q_{r}\left(u_{1}, u_{2}, \cdots\right)$ and $u_{j}=\operatorname{tr} L_{i}\left(A^{j}\right)$. Here $X$ indicates Kronecker direct product and $S_{r}(A)$ (the symmetrized Kronecker $r$ th power) describes the way ordinary monomials transform under the linear transformation $A$ on the variable.

\section{Lemma II. $\sum \phi(\alpha)=\sum[(\alpha)]$.}

It was proved $\left[7\right.$, p. 386] in FR that $\sum[(\alpha)]=s_{1}^{m}$, where the summation runs over all partitions $\alpha_{1}+2 \alpha_{2}+\cdots+m \alpha_{m}=m$ of $m$. In Lemma I, it was proved that $\sum \phi(\alpha)=s_{1}^{m}$. Therefore the lemma follows.

To prove the theorem, make the induction hypothesis that $t_{1}(g ; s)=[g]$ $=\operatorname{tr} L_{g}(A)$ for all $g<m$. This carries with it the hypothesis that $t_{h}(g ; s)$ $=\operatorname{tr} L_{g}\left(A^{h}\right)$ for all $g<m$. Therefore $q_{\alpha_{g}}(t(g ; s))=q_{c_{g}}\left(\operatorname{tr} L_{g}(A)\right)$ for all $g<m$ so that for any partition $(\alpha)$ where $\alpha_{m}=0,[(\alpha)]=\phi(\alpha)$. Combining this fact with $\sum_{(\alpha)} \phi(\alpha)=\sum_{(\alpha)}[(\alpha)]$ and cancelling all $\phi(\alpha),[(\alpha)]$ with $\alpha_{m}=0$, we have left $\phi(m)=[m]$, that is, $t_{1}(m ; s)=[m]$, which completes the procf of the theorem.

COROLLARY I. If $m$ is a prime number, the coefficients $a(\lambda)$ in $[m]=\sum_{\lambda} a(\lambda)\{\lambda\}$. are the "nearest" integral coefficients of the characters of the unit class $\chi_{(1 m)}^{(\lambda)} d i$ vided by $m$.

Proof. If $m$ is a prime number, the coefficients of $s_{1}^{m}$ and $s_{m}$ in the character formula proved above will be +1 and -1 respectively since $\mu(1)=1$ and $\mu(m)=(-1)^{k}$ if $m$ is a product of $k$ distinct primes greater than 1 . For any partition $(\alpha): \alpha_{1}+2 \alpha_{2}+\cdots+m \alpha_{m}=m$, set $s_{(\alpha)}=s_{1}^{\alpha_{1}} s_{2}^{\alpha_{2}} \cdots s_{m}^{\alpha_{m}}$. Then [1, p. 86] $s_{(\alpha)}=\sum_{\lambda} \chi_{(\alpha)}^{(\lambda)}\{\lambda\}$. But [2, p. 134] the characters of the class containing but one cycle on $m$ letters are given by the formula

$$
\left\{m-k+1,1^{k-1}\right\}_{\alpha_{m}=1}=(-1)^{k-1}, \quad k=1,2, \cdots, m \text {; all other }\{\lambda\}_{\alpha_{m}}=0 \text {. }
$$

Therefore the amount contributed to the total sum by the class containing 
but one cycle on $m$ letters will always be equal to $1,-1$, or 0 . This is not sufficient to add one or subtract one from the coefficient for all cases where $m$ is a prime greater than 2. Direct computation suffices in the case of 2. Even in the case of non-primes, the coefficients of the unit class are the predominating ones.

Character tables $[1 ; 9 ; 10 ; 5]$ for the symmetric group $\Im_{m}$ are published only for $m \leqq 14$. Using these and the above theorem we have computed the multiplicities of the irreducible constituents of the $m$ th Lie representation for $m \leqq 14$. Because of their length, the tables of these numbers are not included in the present paper. Those for $m \leqq 10$ are given [7, pp. 387-388] in $\mathrm{FR}$. The result for [10] in FR is incorrect. The correct result is:

$$
\begin{aligned}
{[10]=} & \{91\}+3\{82\}+4\left\{81^{2}\right\}+8\{73\}+16\{721\}+8\left\{71^{3}\right\}+8\{64\} \\
& +32\{631\}+21\left\{62^{2}\right\}+36\left\{621^{2}\right\}+12\left\{61^{4}\right\}+5\left\{5^{2}\right\}+29\{541\} \\
& +46\{532\}+55\left\{531^{2}\right\}+53\left\{52^{2} 1\right\}+45\left\{521^{3}\right\}+13\left\{51^{5}\right\}+23\left\{4^{2} 2\right\} \\
& +32\left\{4^{2} 1^{2}\right\}+22\left\{43^{2}\right\}+77\{4321\}+52\left\{431^{3}\right\}+28\left\{42^{3}\right\} \\
& +58\left\{42^{2} 1^{2}\right\}+34\left\{421^{4}\right\}+9\left\{41^{6}\right\}+20\left\{3^{3} 1\right\}+27\left\{3^{2} 2^{2}\right\}+44\left\{3^{2} 21^{2}\right\} \\
& +24\left\{3^{2} 1^{4}\right\}+29\left\{32^{3} 1\right\}+31\left\{32^{2} 1^{3}\right\}+16\left\{321^{5}\right\}+3\left\{31^{7}\right\}+3\left\{2^{5}\right\} \\
& +10\left\{2^{4} 1^{2}\right\}+7\left\{2^{3} 1^{4}\right\}+4\left\{2^{2} 1^{6}\right\}+\left\{21^{8}\right\} .
\end{aligned}
$$

3. Generating idempotents of right ideals of symmetry operators. The intersection of $L^{m}$ with any characteristic ideal is a subspace of $L^{m}$ which is invariant under linear automorphisms. In the study of characteristic ideals it is important to know relationships between $L^{m}$ and $L^{m+1}$. In this section we study the nature of the irreducible invariant subspaces of $L^{m}$. If we know $I \cap L^{m}\left(=I^{m}\right)$, then there are certain necessary conditions imposed upon $I \cap L^{m+1}\left(=I^{m+1}\right)$.

Let $P_{m}$ denote the vector space of dimension $n^{m}$ composed. of tensors of rank $m$. In FR $\left[7\right.$, pp. 384-385] it was shown that $L^{m}$ is a factor space of $P_{m}$. Let $\mu\left(i_{1}, \cdots, i_{m}\right)$ be a set of basis tensors of $P_{m}$ for which

$$
\begin{aligned}
A \mu\left(i_{1} \cdots i_{m}\right) \rightarrow \mu^{\prime}\left(i_{1} \cdots i_{m}\right) & =\sum \alpha_{i k} \mu\left(k_{1} \cdots k_{m}\right) \\
& =\sum \alpha_{i_{1} k_{1}} \cdots \alpha_{i_{m} k_{m}} \mu\left(k_{1} \cdots k_{m}\right) .
\end{aligned}
$$

Set $\sigma_{m} \mu\left(i_{1} \cdots i_{m}\right)=r_{i_{1}} \ldots i_{m}$ and extend $\sigma_{m}$ into a mapping of the whole of $P_{m}$ onto $L^{m}$ by linearity. We call $\sigma_{m}$ the natural mapping and denote the kernel of the mapping by $Q_{m}$ and call $Q_{m}$ the space of relations.

TheOREM IV. The natural mapping $\sigma_{m}$ of the tensor space $P_{m}$ on the Lie space $L^{m}$ is an operator homomorphism under the operators $A$, that is, if $\gamma$ is a tensor of $P_{m}$ and $l$ is an element of $L^{m}$, then if $\sigma_{m} \gamma=l, \sigma_{m} A \gamma=A l$ and the space of relations $Q_{m}$ is an invariant subspace of $P_{m}$.

Proof. It is sufficient to consider basis tensors. 


$$
\sigma_{m} A \mu\left(i_{1} \cdots i_{m}\right)=\sum \alpha_{i_{1} k_{1}} \cdots \alpha_{i_{m} k_{m}} r_{k_{1}} \cdots k_{m}
$$

by the definition of $\sigma_{m}$.

$$
\begin{aligned}
A \sigma_{m} \mu\left(i_{1} \cdots i_{m}\right)=A r_{i_{1}} \cdots i_{m} & =A f_{i}^{m}\left(r_{i_{1}} \cdots i_{m}\right) \\
& =f_{i}^{m} \sum \alpha_{i_{1} k_{1}} r_{k_{1}} \cdots \sum \alpha_{i_{m} k_{m}} \boldsymbol{r}_{k_{m}} \\
& =\sum \alpha_{i_{1} k_{1}} \cdots \alpha_{i_{m} k_{m}} \boldsymbol{r}_{k_{1}} \cdots k_{m} .
\end{aligned}
$$

Therefore $\sigma_{m} A \mu\left(i_{1} \cdots i_{m}\right)=A \sigma_{m} \mu\left(i_{1} \cdots i_{m}\right)$ and thus $\sigma_{m}$ is an operator homomorphism under the operators $A$. Since $Q_{m}$ was defined as the set of elements of $P_{m}$ which map into zero under $\sigma_{m}$, it follows that $\sigma_{m} A Q_{m}=0$ for all $A$. Hence $Q_{m}$ is invariant under $A$, since every tensor of $Q_{m}$ is carried into a tensor of the same subspace.

Consider symmetry operators $\left[4\right.$, p. 97] $a$ acting on the tensor space $P_{m}$. Consider all such $a$ so that $a P_{m} \subseteq Q_{m}$. Since $Q_{m}$ is an invariant subspace of $P_{m}$, it will be characterized by these $a$ 's. If $a$ has the property that $a P_{m} \subseteq Q_{m}$, so does $a b$, since $(a b) P_{m}=a\left(b P_{m}\right) \subseteq a P_{m} \subseteq Q_{m}$. Hence, the set of all such $a$ 's is a right ideal $J_{m}$ in the group ring $R_{m}$ of the symmetric group. Weyl shows $[4$, p. 97] that, considered abstractly, these symmetry operators form the group ring of the symmetric group, provided $n \geqq m$. We can characterize right ideals in the semi-simple ring by means of some generating idempotent. This idempotent is not unique however. Let $\mathfrak{S}_{1}$ denote the permutation group on the first letter, $\Im_{2}$ the permutation group on the first two letters, and so on. Then imbed one symmetric group in the succeeding one so that $\Im_{1} \subset \Im_{2} \subset \ldots$ $\subset \Im_{m}$. The group rings are then imbedded in each other and the right ideal $J_{m-1} \subset R_{m-1}$. It is obvious that $J_{m-1} R_{m} \subset J_{m}$ and hence $J_{i} \subset J_{p}$ for $p>i$, that is, if $\sum_{i} a\left(i_{1} \cdots i_{m-1}\right) F\left(i_{1} \cdots i_{m-1}\right) \in Q_{m-1}$ then $\sum_{i} a\left(i_{1} \cdots i_{m-1}\right) F\left(i_{1} \cdots i_{m-1} k\right)$ $\in Q_{m}$ for $k=1, \cdots, n$. This we call the hereditary property.

Let $e_{m}$ denote an idempotent of $J_{m}$ such that $e_{m} R_{m}=J_{m}$. We are interested on the one hand in the computation of generating idempotents for the right ideals of symmetry operators of $P_{m}$ and on the other hand in a sufficient set of relations connecting right normed products. We want an idempotent generator for the ideal such that $e_{m} P_{m}=Q_{m}$. Since $J_{m-1} R_{m} \subset J_{m}$, we can proceed inductively and choose $e_{m}=e_{m-1}+e_{m}^{\prime}$ where $e_{m-1}$ and $e_{m}^{\prime}$ are orthogonal idempotents.

Using the Jacobian identity $\left(^{(5)}\right.$ of the free Lie ring, we get relations among the generators and thus obtain symmetry operators which, when applied to the tensors of $P_{m}$, map them onto $Q_{m}$. The following two properties are useful in the computation:

$$
\begin{gathered}
\left(r_{1} \circ r_{2}\right) r_{i} \ldots_{n}=r_{i} \ldots n 21 \\
f_{i}^{s}\left(r_{1} \circ r_{i}, \cdots n 12,\right. \\
\left.r_{2}, \cdots, r_{s+1}\right)+f_{i}^{s}\left(r_{2} \circ r_{1}, \cdots, r_{s+1}\right)=0,
\end{gathered}
$$

since $f_{i}^{s}\left(r_{1} \circ r_{2}+r_{2} \circ r_{1}, \cdots, r_{s+1}\right)=f_{i}^{s}\left(0, r_{3}, \cdots, r_{s+1}\right)=0$.

(5) $a(b c)+b(c a)+c(a b)=0$. 
In the computation of the generating idempotents of $J_{m}$, we make use of seminormal idempotents [8, pp. 611-614] which, along with their corresponding matrix basis units, were designed to handle the case of imbedding one symmetric group in another. From the analysis of the Lie representation made in $\$ 2$, we know when we have enough generators to fill $J_{m}$. The regular Young diagrams $[8$, p. 611] belonging to the partition $(\lambda)$ of $m$ are numbered according to their dictionary order $[8, \mathrm{p} .611]$. By application of the hereditary property and by the analysis of the $m$ th Lie representation calculated in $\$ 2$, we are able to determine whether a computation showing the relationship between idempotents is necessary. Let us, for example, compute a generating idempotent of $J_{5}$. All of the diagrams either belong to the Lie space or belong to the space of relations on account of the hereditary property, except two diagrams in the partition $\left(31^{2}\right)$, where we learn from the analysis of the 5 th Lie representation that one belongs to the Lie space. Hence there must exist some relationship between the primitive idempotents corresponding to these two diagrams. This relationship we seek to find.

In order to find a relation among the $r_{i}$ 's which involves all indices of $r_{12345}$, we make use of the Jacobian identity, letting $a=r_{12}, b=r_{3}, c=r_{45}$. By repeated use of Property (1), page 534, we have $r_{12345}-r_{12354}+r_{45321}-r_{45312}$ $+r_{12543}-r_{12453}=0$ or $[1-(45)+(1524)-(14)(25)+(35)-(354)] r_{12345}=0$. Writing this as a product of consecutive transpositions and multiplying the matrices $\left[8\right.$, p. 612] corresponding to the transpositions $t_{r}$, we note in the final matrix rows three and five, since the diagrams which did not belong to the relation space or which we could not clearly identify as belonging to the Lie space are the diagrams

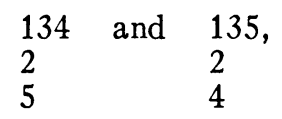

which are the third and fifth respectively in the partition $\left(31^{2}\right)$. Denote by $e_{m}$ the generating idempotent of $J_{m}$ and denote by $\boldsymbol{e}_{t t^{\prime}}$ the product of the seminormal idempotents corresponding to the $t$ th and $t^{\prime}$ th regular Young diagrams in a particular partition of $m$. From the final matrix we conclude that $5 / 2 e_{33}-(15)^{1 / 2} / 2 e_{35}-5(15)^{1 / 2} / 6 e_{53}+5 / 2 e_{55}$ is in the ideal. Normalizing we obtain $e_{33}-(15)^{1 / 2} / 3 e_{53}$ so that we may take $e_{5}=e_{4}+e_{33}-(15)^{1 / 2} / 3 e_{53}$. Here $e_{4}$ and $e_{33}-(15)^{1 / 2} / 3 e_{53}$ are orthogonal idempotents. The following are generating idempotents of $J_{m}, m \leqq 6$, where the regular Young diagram to which the semi-normal idempotent corresponds is written out in full.

$$
\begin{aligned}
& e_{1}=0 ; \quad e_{2}=e_{1}+e_{12} ; \quad e_{3}=e_{2}+\underset{\substack{2 \\
3}}{e_{1} ;} \quad e_{4}=e_{3}+\underset{24}{e_{13}}
\end{aligned}
$$

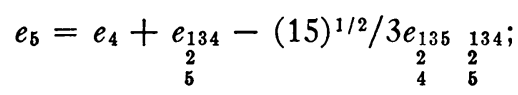




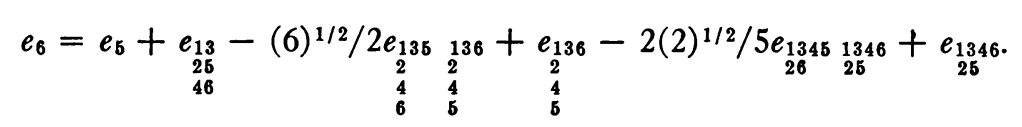

In case $m=7$, computations to find the relationships among the idempotents involve matrices of degree 35 . Hence it seems impractical to carry this work further until a better method is established.

With the information of this section, if we limit ourselves to linear automorphisms, it is an easy matter to determine $I^{m}=I \cap L^{m}$ for $m \leqq 6$. In the case of even quadratic automorphisms, the problem becomes much more complicated. Certain information concerning the free Lie ring over a field of characteristic zero has been obtained. Certain necessary and certain sufficient conditions that a given ideal be characteristic have been given. The determination of all of the characteristic ideals of the free Lie ring remains an unsolved problem.

\section{BIBLIOGRAPHY}

1. D. E. Littlewood, The theory of group characters and matrix representations of groups, Oxford, Clarendon Press, 1940.

2. F. D. Murnaghan, The theory of group representations, Baltimore, The Johns Hopkins Press, 1938. 1939.

3. J. V. Uspensky and M. A. Heaslet, Elementary number theory, New York, McGraw-Hill,

4. H. Weyl, The classical groups, Princeton, Princeton University Press, 1939.

5. K. Kondo, Tables of characters of the symmetric group of degree 14, Proceedings of the Physics-Mathematical Society of Japan (3) vol. 22 (1940) pp. 585-593.

6. W. Magnus, Über Beziehungen zwischen hoheren Kommutatoren, J. Reine Angew. Math. vol. 177 (1937) pp. 105-115.

7. R. M. Thrall, On symmetrized Kronecker powers and the structure of the free Lie ring, Amer. J. Math. vol. 64 (1942) pp. 371-388.

8. - Young's semi-normal representation of the symmetric group, Duke Math. J. vol. 8 (1941) pp. 611-624.

9. M. Zia-ud-Din, The characters of the symmetric group of order 11!, Proc. London Math. (2) vol. 39 (1935) pp. 200-204.

10. - The characters of the symmetric group of degrees 12 and 13, Proc. London Math.. Soc. (2) vol. 42 (1937) pp. 340-355.

WhEATON COLLĖGE, WhEATON, ILL. 